\title{
Quais as evidências científicas sobre Auriculoterapia e a COVID-19?
}

\author{
What is the scientific evidence on Auriculotherapy and COVID-19? \\ ¿Cuál es la evidencia científica sobre Auriculoterapia y COVID-19?
}

\author{
Daniela Aparecida de Faria \\ ORCID: https://orcid.org/0000-0001-8938-9371 \\ Centro Universitário UNA, Brasil \\ E-mail: danielaffisio@hotmail.com \\ Anna Laura Oliveira Aragão Ferreira \\ ORCID: https://orcid.org/0000-0001-5020-3402 \\ Centro Universitário UNA, Brasil \\ E-mail: annalauraaragao99@gmail.com \\ Kelly Aline Rodrigues Costa \\ ORCID: https://orcid.org/0000-0003-4289-1780 \\ Centro Universitário UNA, Brasil \\ E-mail: kellyalinerodrigues@yahoo.com.br \\ Leidna Oliveira Melo \\ ORCID: https://orcid.org/0000-0003-4083-1549 \\ Centro Universitário UNA, Brasil \\ E-mail: leidnao@gmail.com \\ Mateus Saldanha Fróis \\ ORCID: https://orcid.org/0000-0001-7835-6523 \\ Centro Universitário UNA, Brasil \\ E-mail: mateussaldanha2011@gmail.com \\ Patrícia Aparecida Tavares \\ ORCID: https://orcid.org/0000-0002-3626-5217 \\ Centro Universitário UNA, Brasil \\ E-mail: tavaresaguiar@yahoo.com.br \\ Nayara Ragi Baldoni \\ ORCID: https://orcid.org/0000-0002-3400-0725 \\ Centro Universitário UNA, Brasil \\ E-mail: nraldoni@gmail.com \\ Stefanie Marina Pimenta Santos \\ ORCID: https://orcid.org/0000-0003-2878-424 \\ Centro Universitário UNA, Brasil \\ E-mail: stefanie.santos3108@gmail.com
}

\section{Resumo}

Este estudo tem por objetivo, reunir e analisar evidências sobre o uso da Auriculoterapia durante a pandemia da COVID-19. Trata-se uma revisão integrativa, realizada nas bases de dados PubMed, Scopus, Virtual Health Library (VHL), Web of Science and Science Direct com estudos publicados até 11/06/2021. Utilizou-se o Guideline Preferred Reporting Items for Systematic Reviewsand Meta-Analyses - The PRISMA Statement. A pergunta da pesquisa foi: Quais as contribuições/atuação da Auriculoterapia durante a pandemia da COVID-19? Foram encontrados um total de 16 estudos sendo que após análise, foram incluídos neste trabalho cinco estudos. Dentre os estudos analisados todos, $100 \%(\mathrm{n}=5)$ apresentaram uma melhora na redução das queixas tratadas e melhora na qualidade dos profissionais de saúde atendidos pela Auriculoterapia. Observou-se que a Auriculoterapia se mostrou eficaz na redução dos quadros de ansiedade, estresse, depressão além de, proporcionar uma melhora no desempenho profissional e na qualidade de sono destes profissionais de saúde em tempos de pandemia COVID-19. O presente estudo evidenciou COVID-19 resultados positivos quanto ao uso da Auriculoterapia. A terapia apresentou eficácia na redução dos quadros de ansiedade, estresse, depressão além de, proporcionar uma melhora no desempenho profissional e na qualidade de sono destes profissionais de saúde, porém estudos futuros e de melhor nível de evidência são necessários para se identificar os resultados do uso da auriculoterapia sob os impactos causados pela pandemia.

Palavras-chave: COVID-19; Coronavírus; Auriculoterapia.

\section{Abstract}

This study aims to gather and analyze evidence on the use of Auriculotherapy during the COVID-19 pandemic. This is an integrative review, carried out in the PubMed, Scopus, Virtual Health Library (VHL), Web of Science and 
Science Direct databases with studies published until 11/06/2021. The Guideline Preferred Reporting Items for Systematic Reviews and Meta-Analyses - The PRISMA Statement was used. The research question was: What are the contributions/action of Auriculotherapy during the COVID-19 pandemic? A total of 16 studies were found, and after analysis, five studies were included in this work. Among the studies analyzed, $100 \%(\mathrm{n}=5)$ showed an improvement in the reduction of complaints treated and an improvement in the quality of health professionals assisted by Auriculotherapy. It was observed that Auriculotherapy proved to be effective in reducing anxiety, stress and depression, in addition to providing an improvement in professional performance and in the quality of sleep of these health professionals in times of the COVID-19 pandemic. The present study showed COVID-19 positive results regarding the use of Auriculotherapy. The therapy was effective in reducing anxiety, stress, depression, in addition to providing an improvement in the professional performance and quality of sleep of these health professionals, but future studies with a better level of evidence are needed to identify the results of use of auriculotherapy under the impacts caused by the pandemic.

Keywords: COVID-19; Coronavirus; Auriculotherapy.

\section{Resumen}

Este estudio tiene como objetivo recopilar y analizar evidencia sobre el uso de Auriculoterapia durante la pandemia de COVID-19. Se trata de una revisión integradora, realizada en las bases de datos PubMed, Scopus, Virtual Health Library (VHL), Web of Science y Science Direct con estudios publicados hasta el 06/11/2021. Elementos de presentación de informes preferidos de la guía para revisiones sistemáticas y metaanálisis: se utilizó la declaración PRISMA. La pregunta de investigación fue: ¿Cuáles son los aportes / acción de la Auriculoterapia durante la pandemia de COVID-19? Se encontraron un total de 16 estudios y, después del análisis, se incluyeron cinco estudios en este trabajo. Entre todos los estudios analizados, el 100\% $(n=5)$ mostró una mejora en la reducción de las quejas tratadas y una mejora en la calidad de los profesionales de la salud asistidos por Auriculoterapia. Se observó que la Auriculoterapia demostró ser efectiva en la reducción de la ansiedad, el estrés y la depresión, además de brindar una mejora en el desempeño profesional y en la calidad del sueño de estos profesionales de la salud en tiempos de la pandemia COVID-19. El presente estudio mostró resultados positivos de COVID-19 con respecto al uso de Auriculoterapia. La terapia fue eficaz en la reducción de la ansiedad, el estrés, la depresión, además de brindar una mejora en el desempeño profesional y la calidad del sueño de estos profesionales de la salud, pero se necesitan estudios futuros con un mejor nivel de evidencia para identificar los resultados del uso de la auriculoterapia bajo los impactos causados por la pandemia.

Palavras clave: COVID-19; Coronavirus; Auriculoterapia.

\section{Introdução}

O novo Coronavírus (SARS-COV-2) é o sétimo já conhecido da família Coronavirida e que infecta seres humanos e é o agente causador da atual pandemia da COVID-19 (Brito et al., 2020; Gasmi et al., 2020; Zhang \& Liu, 2020). Segundo a Organização Mundial de Saúde (OMS) os EUA, Índia e Brasil são países com maior número de casos (Brasil, 2021; WHO, 2020).

A COVID-19 pode afetar vários sistemas no corpo humano e possui uma variação de sintomas que vão desde efeitos físicos leves, como tosse, febre, dispneia, mialgia, artralgia, fadiga, diarreia e cefaleia. A infecção causada pelo SARS-CoV-2 pode evoluir para distúrbios e deficiências de múltiplos órgãos como os rins, fígado, coração e cérebro e a Síndrome de Angústia Respiratória do Adulto-SDRA que é a mais comum das complicações pela infecção observada em casos de urgência e emergência, e frequentemente evoluindo para tratamento em Unidades de Terapia Intensiva (UTI) e que pode evoluir para óbito (OPAS, 2020; WHO, 2020). Alguns efeitos psíquicos como aumento do nível de estresse, raiva, ansiedade, culpa, tristeza, depressão, distúrbios do sono, suicídio, ataques de pânico generalizado e agressividade são os efeitos psíquicos estão correlacionados com o distanciamento social, insegurança e incertezas impostos pela doença (Pereira et al, 2020; Silva et al., 2020).

A pandemia impactou negativamente na qualidade de vida dos pacientes e dos profissionais de saúde uma vez que, resultou no aumento da demanda nos serviços de saúde de todo mundo, a introdução de equipamentos de proteção individual e alteração em todo o ambiente de trabalho (Kerins et al., 2021). Além disso, profissionais da saúde convivem ainda o medo de infectar outras pessoas principalmente da família e vivenciam diariamente o contágio e até morte de colegas (Trigueiro et al., 2020). 
Diante desse cenário em que os pacientes se apresentam com sequelas por longos períodos e trabalhadores de saúde estão em estados de sofrimento psíquico torna-se importante ferramentas que ajudem ao enfretamento desta condição. A Medicina Tradicional Chinesa (MTC) por meio da Auriculoterapia pode ser uma importante abordagem complementar ao processo de reabilitação destes indivíduos. Trata-se de uma técnica milenar que possui como característica principal o olhar sobre o ser humano como um todo e quando o corpo está em um estado de harmonia e equilíbrio, ele está saudável (Artioli et al., 2019). Portanto, conhecer os resultados que esta técnica pode contribuir para a saúde de indivíduos é grande relevância. Considerando o exposto o objetivo deste estudo é reunir e analisar evidências sobre o uso da Auriculoterapia durante a pandemia da COVID-19.

\section{Metodologia}

Reuniu-se evidências sobre a COVID-19 e o uso de Auriculoterapia. Para isso, utilizou-se o Guideline Preferred Reporting Items for Systematic Reviewsand Meta-Analyses - The PRISMA Statement (Moher et al., 2009). A pergunta da pesquisa foi: Quais as contribuições/atuação da Auriculoterapia durante a pandemia da COVID-19? Foram considerados elegíveis todos os estudos encontrados na literatura sem limite de tempo que descreveram o uso da Auriculoterapia durante o período da pandemia. Foram excluídos os artigos de revisão, os editoriais, as cartas ao editor, as notícias e comentários.

A busca de evidência foi realizada em cinco diferentes bases, sendo elas: PubMed, Scopus, Biblioteca Virtual de Saúde (BVS), Web of Science e Science Direct com estudos publicados até 11/06/2021, sem restringir data de início da coleta e idioma, uma vez que o objetivo foi recuperar o máximo de artigos possíveis. Para a elaboração da estratégia de busca utilizou o MeSH e os Descritores em Saúde (Desc) das palavras "COVID-19"/"Infecções por Coronavirus" e "Auriculotherapy" associados com os operadores booleanos "AND" e "OR".

Para classificar o nível de evidência dos artigos, foi utilizada a categorização da Agency for Health care Research and Quality, segundo a qual o nível 1 é considerado o de maior força de evidência, no qual são incluídas as metanálises de múltiplos estudos controlados. Projetos individuais com desenho experimental, como os ensaios clínicos aleatórios, são considerados de nível 2. Estudos de coorte, caso-controle e quase experimentais, como estudos não randomizados, são classificados como nível 3. Estudos com desenho não experimental, como os transversais, recebem o nível de evidência 4. Relatórios de caso são considerados nível 5, e opiniões de autoridades respeitáveis baseadas na competência clínica ou opinião de comitês de especialistas e interpretações de informações não baseadas em pesquisas estão no nível 6.

A seleção e leitura dos artigos foram realizadas por dois autores, MSF e ALOAF em caso de divergência um terceiro autor entrou para dar o consenso (DAF). Após a seleção dos artigos que seriam incluídos coletou-se as seguintes variáveis: autores, ano publicação, delineamento do estudo, país, tamanho amostral, instrumento e dados das sessões, principais resultados, nível de evidência do estudo.

\section{Resultados e discussão}

A pesquisa com os descritores nas bases de dados resultou em um total de 16 artigos, destes, dois eram duplicados. Em seguida, realizou-se leitura na íntegra de 14 artigos sendo nove excluídos segundo os critérios de elegibilidade. Por fim, foram incluídos no estudo 5 artigos (Figura 1). O resultado desta investigação encontra-se na Tabela 1.

Dentre os estudos analisados todos, $100 \%(\mathrm{n}=5)$ observou-se uma melhora na redução das queixas tratadas e melhora na qualidade dos profissionais de saúde atendidos pela Auriculoterapia. Em relação ao nível de evidência dos estudos $40 \%(\mathrm{n}=2)$ foram relatos de caso e estudo de caso com nível 5 , seguidos de $40 \%(\mathrm{n}=2)$ de estudos quasiexperimentais com nível de evidência 3 e $(n=1)$ e estudo observacional com nível de evidência 4 . A maioria dos estudos 
eram brasileiros $80 \%(\mathrm{n}=4)$ e um dos estudos foi conduzido na Espanha $20 \%(\mathrm{n}=1)$. No que se refere ao tamanho amostral dos estudos, houve uma variação de 22 a 48 pacientes, sendo que, todos os participantes eram profissionais de saúde.

O tempo de acompanhamento das sessões variaram de 2 semanas há 6 semanas, com variância de 2 a 6 sessões no total, quanto a frequência das sessões variaram de 5 dias a 7 dias, sendo de maior ocorrência em 7 dias $(n=4)$, com duração média de 8 a 20 minutos por sessão. Quanto aos pontos auriculares estimulados houve uma variação desde: Shenmen, Rim, Sistema Nervoso Autônomo, Pulmão, Baço, Sistema Nervoso Simpático (SNS), Alegria, Ansiedade, Antidepressivo, Coração, Endócrino, Relaxamento Muscular, Subcórtex, Ponto zero ou Relaxamento muscular e Suprarrenal, Ponto de Valium, tronco cerebral, Yang 1 e Yang 2. O pulmão 1 e 2, shenmen, coração, occipital, ping chuan e baço tem uma ação nos comprometimentos cardiorrespiratórios como o cansaço e a dispneia, e os pontos shen men, baço, analgésico, ponto zero, fígado, baço, tálamo e hipófise estão relacionadas as alterações musculoesqueléticas e neurológicas (Artioli et al., 2019; Morais et al., 2020; Garcia, 1999), deste modo a auriculoterapia poderia ter um efeito positivo nas complicações físicas do COVID-19 e deste modo, melhorar as atividades de vida diárias e limitações funcionais.

Dentre os pontos citados, os pontos de maior ocorrência foram: Shenmen, Rim, Sistema Nervoso Autônomo, Pulmão, Sistema Nervoso Simpático, Coração, Ansiedade, Endócrino e Relaxamento muscular. No que se refere aos instrumentos utilizados não houve um consenso, porém o de predominância foi a escala Despression, Anxiety and Stress Scalae para mensurar o estado de ansiedade, depressão e estresse (DASS-21) em $40 \%(\mathrm{n}=2)$. Seguido do Índice da Qualidade do Sono de Pittsburg (PSQI) $(\mathrm{n}=1)$, outro $(\mathrm{n}=1)$ utilizou análise de conteúdo de Bardi baseado na Teoria do Conforto de Katharine Kolcaba e um estudo não mencionou o instrumento utilizado. Dentre os principais resultados encontraram melhora da qualidade e duração do sono, eficiência habitual, diminuição no uso de medicamentos para dormir; houve melhora no fortalecimento do vínculo entre os profissionais, melhor ambiência e desempenho dos profissionais, empoderamento e cooperação entre os profissionais e redução no estresse, ansiedade e depressão dos profissionais.

A partir dos estudos encontradas foi possível observar que as evidências sobre o uso da Auriculoterapia ainda são escassas, apenas cinco publicações sobre esta temática foram encontradas. Também se notou que os tipos estudos são de baixo nível de evidência e com amostras pequenas. Estes achados já eram esperados, visto que, a pandemia da Covid19 é recente e estudos mais robustos não teria tempo hábil. 
Figura 1. Fluxograma da pesquisa: identificação, seleção, elegibilidade inclusão dos artigos científicos, de acordo com guideline PRISMA

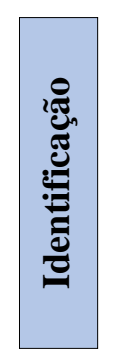

\section{Estudos identificados nas bases de} dados eletrônicos

$$
(n=16)
$$

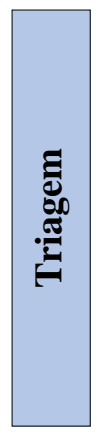

Artigos após retirada da duplicação

$$
(\mathrm{n}=2)
$$

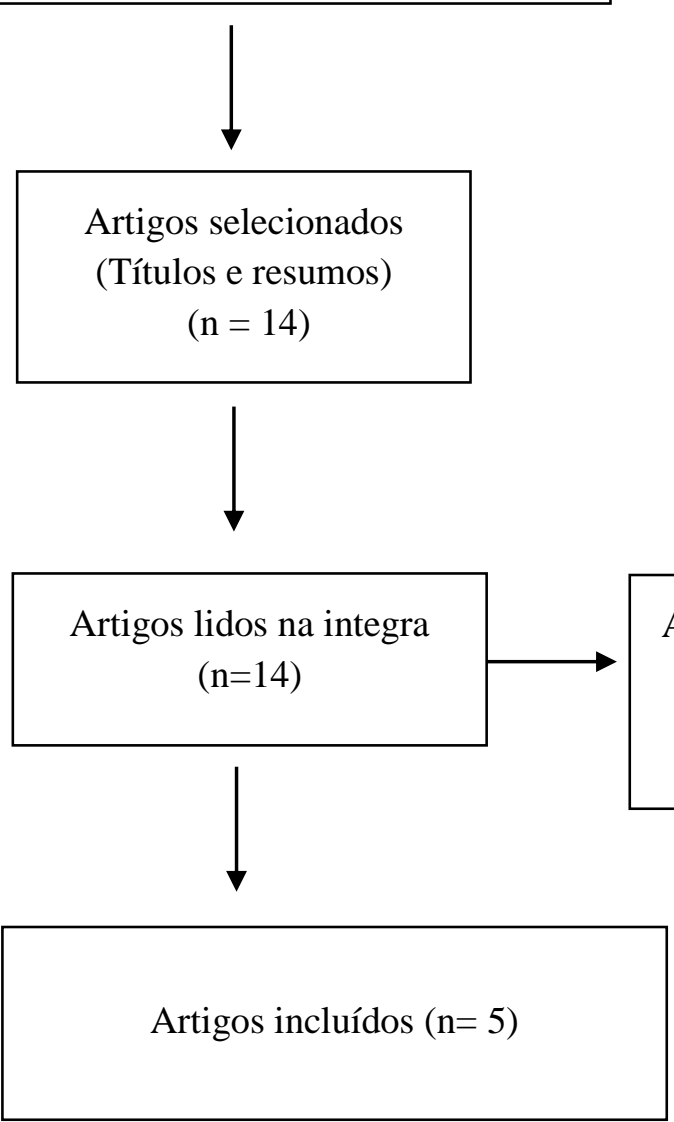

Fonte: adaptado por Moher et al. (2009).

Artigos excluídos segundo critérios de elegibilidade

$$
(\mathrm{n}=9)
$$


Research, Society and Development, v. 11, n. 1, e13711124551, 2022

(CC BY 4.0) | ISSN 2525-3409 | DOI: http://dx.doi.org/10.33448/rsd-v11i1.24551

Tabela 1. Informações e características dos estudos incluídos (n=5).

\begin{tabular}{|c|c|c|c|c|c|c|c|}
\hline Autores & $\begin{array}{c}\text { Delineamento } \\
\text { do estudo }\end{array}$ & $\begin{array}{c}\text { País / } \\
\text { Tamanho } \\
\text { amostral } \\
\end{array}$ & $\begin{array}{c}\text { População } \\
\text { de estudo }\end{array}$ & Instrumento & Dados das sessões & Principais resultados & $\begin{array}{c}\text { Nível de } \\
\text { evidencia }\end{array}$ \\
\hline $\begin{array}{c}\text { Melo et } \\
\text { al., } 2020\end{array}$ & $\begin{array}{l}\text { Estudo quasi- } \\
\text { experimental }\end{array}$ & $\begin{array}{l}\text { Brasil } \\
\mathrm{n}=26\end{array}$ & $\begin{array}{l}\text { Profissionais } \\
\quad \text { de } \\
\text { Enfermagem }\end{array}$ & $\begin{array}{l}\text { O instrumento utilizado para coleta de dados } \\
\text { foi subdividido em: avaliação } \\
\text { sociodemográfica e qualidade do sono. A } \\
\text { qualidade do sono foi mensurada pelo Índice } \\
\text { da Qualidade do Sono de Pittsburg (PSQI). }\end{array}$ & $\begin{array}{l}\text { Realizou-se três sessões de auriculoacupuntura, uma } \\
\text { vez por semana, com duração de } 20 \text { minutos, durante } \\
\text { quatro semanas consecutivas. } \\
\text { Foram estabelecidos os seguintes pontos auriculares: } \\
\text { Shenmen, Rim, Autonômico simpático, Coração, } \\
\text { Pulmão, Subcórtex, Endócrino, Ponto zero ou } \\
\text { Relaxamento muscular e Suprarrenal. }\end{array}$ & $\begin{array}{l}\text { Houve melhora da qualidade e duração do sono, } \\
\text { eficiência habitual, redução nas alteraços do sono e no } \\
\text { uso de medicamentos para dormir. Observou-se } \\
\text { melhora na qualidade do sono de acordo com o PSQI. }\end{array}$ & Nível 3 \\
\hline $\begin{array}{c}\text { Melo et } \\
\text { al., } 2020\end{array}$ & $\begin{array}{c}\text { Estudo } \\
\text { descritivo } \\
\text { qualitativo }\end{array}$ & $\begin{array}{l}\text { Brasil } \\
\mathrm{n}=33\end{array}$ & $\begin{array}{l}\text { Profissionais } \\
\text { de } \\
\text { Enfermagem }\end{array}$ & $\begin{array}{l}\text { Foi utilizado análise de Bardain por meio da } \\
\text { Teoria do Conforto de Katharine Kolcaba para } \\
\text { categorização dos resultados }\end{array}$ & $\begin{array}{l}\text { Foram realizadas } 4 \text { sessões de auriculoacunputura } \\
\text { uma vez por semana, não mencionou os pontos } \\
\text { utilizados nas sessões. } \\
\text { Foi utilizado um questionário estruturado, contendo } \\
\text { questões sócioprofissionais e de identificação de } \\
\text { problemas potenciais relacionados ao conforto e seus } \\
\text { domínios físico, psicoespiritual, sociocultural e } \\
\text { ambiental. }\end{array}$ & $\begin{array}{l}\text { Os resultados foram divididos } \mathrm{m} \text { subcategorias: } \\
\text { Auriculoacupuntra como medida de conforto; } \\
\text { conhecendo e experimentado a auriculoacupuntura; } \\
\text { Expressão de alívio e tranquilidade como níveis de } \\
\text { conforto; (Des)Conforto físico e psicoespiritual e a } \\
\text { atuação na assistência à COVID-19; Sensações de } \\
\text { desconforto percebidas durante a atuação na pandemia; } \\
\text { conforto físico e psicoespiritual melhorados com a } \\
\text { auriculoacupuntura; apoio organizacional ao } \\
\text { comprometimento individual com a saúde; Integridade } \\
\text { institucional; Comportamento de procura de saúde. }\end{array}$ & Nível 4 \\
\hline $\begin{array}{l}\text { Trigueiro } \\
\text { et al., } \\
2020 .\end{array}$ & $\begin{array}{l}\text { Relato de } \\
\text { experiência }\end{array}$ & $\begin{array}{l}\text { Brasil } \\
\mathrm{n}=48\end{array}$ & $\begin{array}{l}\text { Profissionais } \\
\text { da Saúde do } \\
\text { Serviço de } \\
\text { atendimento } \\
\text { móvel de } \\
\text { urgência } \\
\text { (SAMU) } \\
\end{array}$ & & $\begin{array}{l}6 \text { sessões de auriculoterapia, com duração de } 8 \\
\text { minutos, realizado } 2 \text { vezes na semana. } \\
\text { Os pontos auriculares foram: Shenmen, Rim, Sistema } \\
\text { Nervoso Autônomo, Pulmão e Baço. }\end{array}$ & $\begin{array}{l}\text { Fortalecimento do vínculo entre os profissionais, } \\
\text { melhor ambiência, melhor desempenho dos } \\
\text { profissionais, melhora do vínculo com a gestão do } \\
\text { local, empoderamento e cooperação entre os } \\
\text { profissionais, além da criação de um projeto piloto de } \\
\text { cuidado ao profissional do SAMU. }\end{array}$ & Nível 5 \\
\hline $\begin{array}{l}\text { Oliveira } \\
\text { et al., } \\
2021\end{array}$ & $\begin{array}{l}\text { Estudo de } \\
\text { Caso }\end{array}$ & $\begin{array}{l}\text { Brasil } \\
\mathrm{n}=41\end{array}$ & $\begin{array}{c}\text { Profissionais } \\
\text { de } \\
\text { Enfermagem }\end{array}$ & $\begin{array}{l}\text { Questionário } \text { de } \\
\text { sociodemográfico e a escala Despression, } \\
\text { Anxiety e Stress Scalae para mensurar o } \\
\text { estado de ansiedade, depressão e estresse } \\
\text { (DASS-21). }\end{array}$ & $\begin{array}{l}\text { Sessão foi realizada a cada } 5 \text { dias, com duração de } 10 \\
\text { minutos, durante } 15 \text { dias de atendimentos } \\
\text { Foram estabelecidos os pontos auriculares: Shenmen, } \\
\text { Rim, Sistema Nervoso Simpático (SNS), Alegria, } \\
\text { Ansiedade, Antidepressivo, Coração, Endócrino, } \\
\text { Pulmão e Relaxamento Muscular. }\end{array}$ & $\begin{array}{l}\text { Antes da sessão de auriculoterapia, o estresse foi } \\
\text { mensurado em grau moderado, enquanto após a sessão, } \\
\text { o grau de estresse mudou para o nível normal. Houve } \\
\text { também queda observada nas medianas quanto a } \\
\text { depressão e ansiedade. Salienta-se também, a mudança } \\
\text { de profissionais com estresse, ansiedade e depressão em } \\
\text { grau moderado a extremamente grave (antes da sessão) } \\
\text { para os níveis baixos e normal (após a sessão). }\end{array}$ & Nível 5 \\
\hline
\end{tabular}


Percebe-se que os profissionais de saúde durante o período pandêmico da COVID-19 apresentam padrões de desgaste tanto físico quanto psicológico. Isso se deve a rápida evolução da pandemia com suas crescentes taxas de transmissibilidade, de casos tanto leves quanto graves, gerando um aumento significativo na intensidade, na demanda e nas atribuições destes profissionais além de, uma sobrecarga emocional constante (Humerez et al., 2020; Pereira et al., 2021). Tal sobrecarga interfere diretamente no desempenho profissional e nas relações sociais. Segundo estudo realizado em trabalhadores do serviço de urgência no período da pandemia de COVID-19, a auriculoterapia por meio de seis aplicações utilizando-se os seguintes pontos: Shenmen, rim. SNV, pulmão e baço se mostrou eficaz na obtenção do fortalecimento de vínculos e na resolutividade do serviço prestado tendo em vista que, tais profissionais após a terapêutica se encontravam com boa qualidade de sono, ausência de dores físicas (Trigueiro et al., 2020).

Os impactos na vida social e no trabalho, não estão associados apenas os profissionais de saúde, mas também com a população geral. Para Miranda (2021), o isolamento e confinamento podem colocar o corpo humano sob uma grande quantidade de coação psiconeuroendócrina, causando alterações neurocognitivas, sintomas fisiopatológicos mensuráveis e efeitos adversos na saúde mental.

O contato direto com paciente infectados pelo Coronavírus a carreta níveis de ansiedade importante nos profissionais de saúde. Podendo ser observado no estudo com 42 profissionais de enfermagem que utilizaram a escala Depression, Anxiety and Stress Scale-21 e realizaram aplicação de auriculoterapia a cada 5 dias durante 15 dias. Tal estudo, identificou redução nos níveis de ansiedade considerados pela escala como graves (14,7\% para 4,9\%) e, extremamente graves $(14,6 \%$ para $2,4 \%)$. (Oliveira et al., 2021). Corroborando com o estudo observacional coorte prospectivo de Abuye e Sánchez-Pérez (2021) no qual, identificou redução nos níveis de ansiedade quando comparou grupo intervenção $(81,8 \%)$ com grupo controle $(63,6 \%)$ e, quando comparou "pré" e "pós" de ambos os grupos. Sendo que no grupo intervenção o valor médio da ansiedade foi estatisticamente menor no "pós" do que no "pré", o que não aconteceu no grupo controle.

O período da pandemia da COVID-19 fez com que, principalmente profissionais da saúde, vivenciassem situações de alto estresse, sobrecargas físicas e mentais, incertezas relacionadas ao futuro pessoal e de todos com quem convive levando ao aumento do risco de adoecimento físico e mental, podemos gerar quadros futuros graves como depressão e/ou crises de ansiedade (Caldas et al., 2021; Oliveira et al., 2021). Portanto, cuidar desses profissionais que estão na linha de frente do setor saúde torna-se de grande relevância. Neste sentido, estratégia como a auriculoterapia é uma ferramenta oportuna, visto que, as evidências apontam sua efetividade, além disso, é uma técnica que pode ser implementada sem demandar muito recurso financeiro.

No que se refere aos aspectos relacionados a percepção de conforto, queixas álgicas ansiedade e qualidade do sono, estudos realizados por Melo et al., (2020) com profissionais da enfermagem nos mostram que é possível observar uma melhora nestes aspectos. Para isso, em seus estudos, a auriculoterapia foi aplicada semanalmente durante 4 semanas, utilizando-se dos seguintes pontos: Shenmen, rim, SNV, coração, pulmão, Subcórtex, endócrino, Ponto zero ou relaxamento e suprarrenal.

O ensaio clínico randomizado realizado por Kurebayashi et al., (2017) com uma equipe de Enfermagem de um Hospital em São Paulo, comparando a eficácia das intervenções com agulhas semipermanentes e semente na redução da ansiedade. Foi realizado dez sessões no qual como resultado obteve diferenças positivas na redução da ansiedade, sendo que no grupo com agulhas semipermanentes obtiveram grande efeito, apresentando 1,08 no índice de Cohen e redução de ansiedade $17 \%$, ainda com as agulhas apresentou uma redução nos níveis de dor em $34 \%$, em relação ao grupo da semente que obteve $24 \%$ na redução da dor. No domínio mental da qualidade de vida apontou $13 \%$ de aumento. Confirma com o estudo fenômeno gráfico de Landgren et al., (2019) no qual foi abordado com enfermeiros, psiquiatras e terapeutas analisando a acupuntura auricular como benéfica para alivio de sintomas psiquiátricos e somáticos, abordando a eficácia da acupuntura na comunicação 
com o paciente, participação e tomada de decisão compartilhada. Portanto, observa-se que é uma técnica já utilizada com os profissionais da saúde com resultados positivos.

Estudo realizado com profissionais da equipe assistencial de enfermagem em um hospital público de ensino, especializado em Oncologia de adultos e idosos (Silva et al., 2021), identificou melhora nos domínios de depressão, estresse, tensão e confusão mental, em relação ao domínio humor foi observado melhora das relações interpessoais com a equipe de trabalho e com os pacientes, alterando a comunicação e a humanização do cuidar. Os alívios encontrados foram decorrentes do ponto Shenmen, realizado seis sessões de auriculoterapia uma vez por semana durante seis meses, cada sessão teve duração de cinco a dez minutos, no qual os pontos aplicados foram Shenmen, Tronco cerebral e Rim com aplicações de cristais radiônicos permanecendo com eles durante seis dias. Em concordância, o estudo de Prado et al., (2018) avaliando especificamente os níveis de estresse com profissionais de enfermagem do Hospital Beneficência Portuguesa de São Paulo, apresentou melhor resultado na redução dos níveis de estresse em $43 \%$ de efeito no tratamento do grupo auriculoterapia contra $26 \%$ do grupo placebo, identificando redução do estresse. Foram realizadas doze sessões duas vezes na semana, utilizando dos pontos Shenmen e Tronco cerebral, terceiro ponto utilizado foi o Sham sendo escolhido ouvido externo e área da bochecha /face. Ambos apresentam melhora nos domínios avaliados.

A presente investigação apresenta como limitação estudos com baixo nível de evidência. E apesar de não termos definido uma população para o nosso estudo, todos os artigos utilizados estão relacionados com profissionais da saúde. Acreditamos que os benefícios encontrados nos artigos analisados poderiam ter sido observados em qualquer população e com a auriculoterapia faz parte das práticas integrativas complementares, pode não ter sido citada diretamente (Zhou et al., 2020; Lin et al., 2021).

Um outro fator que poderia estar relacionado com o número baixo de publicações é limitação na aplicação da técnica. Acreditamos também que se os benefícios da técnica, tivessem sido experimentados nas alterações físicas decorrentes das complicações causadas pela contaminação com o vírus, os números de estudos poderiam ser maiores. O pulmão 1 e 2, coração, tem uma ação nos comprometimentos cardiorrespiratórios como o cansaço e a dispneia, e os pontos Shenmen, baço, analgésico, ponto zero, estão relacionadas as alterações musculoesqueléticas e neurológicas (Artioli et al., 2019; Morais et al., 2020), deste modo a auriculoterapia poderia ter um efeito positivo nas complicações físicas do COVID-19 e deste modo, melhorar as atividades de vida diárias e limitações funcionais.

Por outro lado, é importante ressaltar que foi realizado uma busca em bases de dados científicas importantes na área da saúde e que a estratégia de busca foi sensível para resgatar o que já tinha sido publicado referente a esta temática. Neste sentido, está investigação reuniu resultados importantes para estudos futuros.

\section{Considerações Final}

O presente estudo evidenciou COVID-19 resultados positivos quanto ao uso da Auriculoterapia. A terapia apresentou eficácia na redução dos quadros de ansiedade, estresse, depressão além de, proporcionar uma melhora no desempenho profissional e na qualidade de sono destes profisionais de saúde. Sabe-se que ainda virão muitos efeitos oriundos da pandemia. Por isso, deve-se ter um olhar atencioso, humanizado voltado para tais profissionais para oferecer terapêuticas eficazes faz parte do processo do cuidar. A Auriculoterapia se mostra relevante devido seus resultados obtidos, por se tratar de uma técnica de baixo custo financeiro, fácil e rápida aplicabilidade.

Estudos futuros e de melhor nível de evidência são necessários para se identificar os resultados do uso da auriculoterapia sob os impactos causados pela pandemia da COVID-19 nos profissionais de saúde, qual melhor protocolo para se utilizar cada situação. E assim, oferecer o melhor a estes profissionais. 


\section{Referências}

Abuye, N. O., \& Sánchez-pérez, I. (2021). Efectividad de la acupuntura y la auriculoterapia para reducir el nível de depresión, ansiedade y estrés em personal sanitário de urgências durante la pandemia de Covid-19. Revista Internacional de Acupuntura; 15(2), 43-50. https://doi.org/10.1016/j.acu.2021.04.001.

Artioli, D. P., Tavares, A. L. F., \& Bertolini, G. R. F. (2019). Auriculoterapia: neurofisiologia, pontos de escolha, indicações e resultados em condições dolorosas musculoesqueléticas: revisão sistemática de revisões. BrJP.; 2(4), 356-61. https://doi.org/10.5935/2595-0118.20190065

Brasil. Ministério da Saúde (2021). Boletim Epidemiológico Especial: Doença pelo Coronavírus COVID-19. Ministério da Saúde, Secretaria de Vigilância em saúde. Versão 1, 04 de Março de 2021. - Brasília

Brito, S. B. P., Braga, I. O., Cunha, C. C., Palácio, M. A. V., \& Takenami, I. (2020). Pandemia da COVID-19: o maior desafio do século XXI. Vigil. Sanit. Debate; 8(2), 54-63. https://doi.org/10.22239/2317-269x.01531.

Caldas, G. R. F., Souza, L. E. S., Nunes, N. A., Alenca, P. R. T. et al. (2021). Novo Coronavírus: impacto na saúde mental dos profissionais de saúde. Research, Society and Development; 10(5), e33910515011.http://dx.doi.org/10.33448/rsd-v10i5.15011.

Garcia, E. G. (1999). Auriculoterapia: Escola Huang Li Chun. Ed. Rocca Ltda.

Gasmi, A., Noor, S., Tippairote, T., Dadar, M., Menzel, A., \& Biorklund, G. (2020). Individual risk management strategy and potential therapeutic options for the COVID-19 pandemic. Clinical Immunology; v.215, 108409. https:// doi.org/10.1016/j.clim.2020.108409.

Humerez, D. C., Ohl, R. I. B., \& Silva, M. C. N. (2020). Saúde mental dos profissionais de enfermagem do Brasil no contexto da pandemia Covid-19: ação do Conselho Federal de Enfermagem. Cogitare Enfermagem; 25, e74115. https://dx.doi.org/10.5380/ce.v25i0.74115.

Kerins, J., Hamilton, A. L., Pringle, J., Falquhar, F., \& Tallentire, V. R. (2021). Exploring the impact of the COVID-19 pandemic on doctors' core workplace needs: a qualitative study of internal medicine trainees in Scotland. BMJ Open; (11): e053506. https://doi.org/10.1136/bmjopen-2021-053506.

Kurebayashi, L. F. S., Turrini, R. N. T., Souza, T. P. B., Marques, C. F., Rodrigues, R. T. F., \& Charlesworth, K. (2017). Auriculotherapy to reduce anxiety and pain in nursing professionals: a randomized clinical trial. Rev. Latino-Am. Enfermagem; 25: e2843. https://doi.org/10.1590/1518-8345.1761.2843.

Landgren, K., Strand, A. S., Ekelin, M., \& Ahlstrom, G. (2019). Ear acupuncture in psychiatric care from the health care prefessionals' perspective: a phenomenographic analysis. Issues in Mental Health Nursing; 40(2), 166-175. https://doi.org/10.1080/01612840.2018.1534908.

Lin, L. W., Ananthakrishnan, A., \& Teerawattananon, Y. (2021). Evaluating traditional and complementary medicine: where do we go from here?. International Journal of Technology Assessment in Health Care; 37, 1-6. https://doi.org/10.1017/S0266462321000179.

Melo, G. A. A., Neto, J. C. G. L., Martins, M. G., Pereira, F. G. F., \& Caetano, J. A. (2020). Benefícios da auriculoterapia em profissionais de enfermagem atuantes na Covid-19 à luz da Teoria do Conforto. Escola Anna Nery; 24(SPE). https://doi.org/10.1590/2177-9465-EAN-2020-0311

Melo, G. A. A., Neto, J. C. G. L., Martins, M. G., Pereira, F. G. F., Caetano, J. A. et al. (2020). Efetividade da auriculoterapia na qualidade do sono de profissionais de enfermagem atuantes na Covid-19. Texto \& Contexto Enfermagem; n. 29:e20200392. https://doi.org/10.1590/1980-265X-TCE-2020-0392.

Miranda, G. B. S. (2021). Fatores associados ao estresse em isolamento social durante a pandemia de Covid-19. Dissertação (Mestrado) - Universidade Federal da Bahia. Instituto de Ciências da Saúde. Programa de em Processos Interativos dos Órgãos e Sistemas. Salvador.

Morais, B. X., Ongaro, J. D., Almeida, F. O., Luz, E. M. F., Greco, P. B. T., \& Magnago, T. S. S. (2020). Auriculoterapia e redução da dor musculoesquelética crônica: revisão integrativa. Rev. Bras. Enferm., 73(6), e20190394. https://dx.doi.org/10.1590/0034-7167-2019-0394.

Moher, D., Liberati, A., Tetzlaff, J., \& Altman, D. G. (2009). Preferred reporting items for systematic reviews and meta- analyses: the PRISMA statement. Ann Intern Med.; 151(7), 264-269. https://doi.org/10.1371/journal.pmed.1000097.

Oliveira, C. M. C., Assis, B. B., Mendes, O. G., Lemos, I. C., Sousa, A. L. C., \& Chianca, T. C. M. (2021). Auriculoterapia em profissionais de enfermagem na pandemia do coronavírus: estudo de casos múltiplos. Rev. Eletr. Enferm.; 23:65678, 1-9. https://doi.org/10.5216/ree.v23.65678.

Oliveira, J. C., Oliveira, S. P., Junior, G. R. S., Silva, L. H. L. et al (2021). O impacto da pandemia de Covid-19 na saúde mental dos profissionais de saúde em município da baixada maranhense. Research, Society and Development; 10(10, e163101018744. http://dx.doi.org/10.33448/rsd-v10i10.18744.

Organização Pan-Americana da Saúde (2020). Alerta epidemiológico complicações e sequelas da COVID-19. Organização Pan-Americana da Saúde; Organização Mundial de Saúde PAHO/WHO. - Washington.

Ouzzani, M., Hammady, H., fedorowicz, Z., \& Elmagarmid, A. (2016). Rayyan-a web and mobile app for systematic reviews. Syst Rev.; 5, 210. https://doi.org/10.1186/s13643-016-0384-4.

Preira, A. C. C., Pereira, M. M. A., Silva, B. L. L., Freitas, C. M. et al. (2021). O agravamento dos transtornos de ansiedade em profissionais de saúde no contexto da pandemia da Covid-19. Brazilian Journal of Health Review; 4(2), 4094-4110.

Pereira, M. D., Oliveira, L. C., Costa, C. F. T., Bezerra, C. M. O., Pereira, M. D., Santos, C. K. A., \& Dantas, E. H. M. (2020). A pandemia de COVID-19, o isolamento social, consequências na saúde mental e estratégias de enfrentamento: uma revisão integrativa. Revista Research, Society and Development; 9(7), 1-35. https://doi.org/10.33448/rsd-v9i7.4548.

Prado, J. M., Kurebayashi, L. F. S., \& Silva, M. J. P. (2018). Auriculoterapia verdadeira e placebo para enfermeiros estressados: ensaio clínico randomizado. Rev. Esc. Enferm. USP; 52:e03334. https://doi.org/10.1590/S1980-220X2017030403334.

Silva, H. G. N., Santos, L. E. S., \& Oliveira, A. K. S. (2020). Efeitos da pandemia no novo Coronavírus na saúde mental de indivíduos e coletividades. J. Nurs. Health; 10, e20104007. 
Research, Society and Development, v. 11, n. 1, e13711124551, 2022

(CC BY 4.0) | ISSN 2525-3409 | DOI: http://dx.doi.org/10.33448/rsd-v11i1.24551

Silva, N. O., Kuba, G., Kurebayashi, L. F. S., \& Turrini, R. N. T. (2021). Efeito da auriculoterapia chinesa sobre o humor de profissionais de saúde: estudo piloto. Rev. Enferm. UFSM; (11), e-53. https://doi.org/10.5902/2179769261883.

Trigueiro, R. L., Araújo, A. L., Moreira, T. M. M., \& Florêncio, R. S. (2020). COVID-19 pandemic: reporto n the use of auriculotherapy to optimize emergency workers' health. Rev. Bras. Enferm.; 73(suppl 2):e20200507. http://dx.doi.org/10.1590/0034-7167-2020-0507.

World Health Organization. (2020). Considerations for quarantine of individuals in the contexto of containment for coronavirus disease (COVID-19): interim guidance. Organização Pan-Americana da Saúde. - Geneva.

Zhang, L., \& Liu, Y. (2020). Potential interventions for novel coronavirus in China: A systematic review.J. Med. Virol.; 92, 479-490. https://doi.org/10.1002/jmv.25707.

Zhou, M., Liang, Q., Pei, Q., Xu, F., \& Wen, H. (2020). Chinese medicine for coronavirus disease 2019 as complementary therapy. A protocol for a systematic review and meta-analysis. Medicine; 99(33). https://doi.org/10.1097/MD.0000000000021024. 